\title{
Simultaneous silencing of XIAP and survivin causes partial mesenchymal-epithelial transition of human pancreatic cancer cells via the PTEN/PI3K/Akt pathway
}

\author{
XIAO-PING YI ${ }^{1 *}$, TONG HAN ${ }^{2 *}$, YI-XIONG LI ${ }^{2}$, XUE-YING LONG ${ }^{1}$ and WEN-ZHENG LI ${ }^{1}$ \\ Departments of ${ }^{1}$ Radiology and ${ }^{2}$ General Surgery, Xiangya Hospital, \\ Central South University, Changsha, Hunan 410008, P.R. China
}

Received March 26, 2014; Accepted January 21, 2015

DOI: $10.3892 / \mathrm{mmr} .2015 .3380$

\begin{abstract}
Pancreatic cancer has one of the highest mortality rates among malignant tumors and is characterized by rapid invasion, early metastasis and chemoresistance. X-linked inhibitor of apoptosis (XIAP) and survivin are two of the most important members of the IAP family. Previous studies have shown that XIAP and survivin were overexpressed in pancreatic cancer and were closely associated with cell proliferation and chemoresistance to gemcitabine. In the present study, stable inhibition of XIAP and survivin in Panc-1 cells was performed using lentivirus-carried short hairpin RNAs. The expression of XIAP, survivin, E-cadherin, Slug, phosphatase and tensin homolog (PTEN) and phosphorylated Akt was then measured. In addition, cell proliferation, apoptosis, invasion and migration were assessed. The results showed that stable inhibition of XIAP and survivin expression in Panc-1 cells significantly reduced cell proliferation, increased apoptosis and partially reversed the epithelial-mesenchymal transition (EMT). Furthermore, the results of the present study demonstrated that the partial reversal of the EMT was accompanied by inhibited cell invasion and migration as well as increased chemosensitivity to gemcitabine in pancreatic cancer cells; this was indicated to be mediated via the PTEN/phosphatidylinositol 3-kinase/Akt signaling pathway. In conclusion, these results suggested that simultaneous inhibition of XIAP and survivin may be a promising strategy for the treatment of pancreatic cancer.
\end{abstract}

Correspondence to: Dr Xiao-Ping Yi, Department of Radiology, Xiangya Hospital, Central South University, 87 Xiangya Road, Changsha, Hunan 410008, P.R. China

E-mail: docteryixiaoping@126.com

${ }^{*}$ Contributed equally

Key words: pancreatic cancer, X-linked inhibitor of apoptosis protein, survivin, invasion, epithelial-mesenchymal transition

\section{Introduction}

Pancreatic cancer (PC) has one of the highest mortality rates amongst malignant tumors as well as the poorest clinical outcome worldwide, with an overall five-year survival rate of $<5 \%$ (1). One of the main reasons for mortality of PC patients is their high potential for rapid invasion and early metastasis (2). Therefore, PC is usually diagnosed at advanced, incurable and metastatic stages of the disease; in addition, only $20 \%$ of patients presenting with localized disease are amenable to surgical resection (3). Furthermore, PC is highly chemoresistant, which also accounts for the low survival rates of patients with PC (3). This outcome suggests that an enhanced understanding of the mechanisms underlying invasion, metastasis and chemoresistance is required for the development of novel therapeutic strategies for the successful treatment of PC.

The ability to evade apoptosis is one of the hallmarks that characterizes tumour cells (4). The inhibitor of apoptosis protein (IAP) family is a group of anti-apoptotic factors in the apoptotic pathway which renders cancer cells insensitive to apoptotic stimulation (5). To date, eight human IAP family members have been identified: X-linked inhibitor of apoptosis (XIAP), cellular IAP 1 (c-IAP-1), c-IAP-2, IAP-like protein 2, livin, neuronal apoptosis inhibitory protein (NAIP), survivin and apollon/bruce. XIAP and survivin are two of the most important members of the IAP family (6); these two factors have been found to be overexpressed in numerous malignant tumors, including pancreatic cancer. In addition, XIAP and survivin have been proposed to be involved in tumor cell proliferation, metastasis and chemoresistance (7-9). Epithelial-mesenchymal transition (EMT) is characterized by the loss of cell-to-cell adhesion and a phenotypic change from an epithelial morphology to a fibroblast-like motile morphology (10). EMT has been reported to have an important role in tumor invasion, metastasis and chemoresistance in diverse solid tumors, including PC (10-12). However, the association between XIAP, survivin and EMT in $\mathrm{PC}$ has remained to be elucidated.

RNA interference (RNAi) technology has been widely used in gene function research and cancer gene therapy (13). In the present study, the expression of XIAP and survivin was stably inhibited using lentivirus-mediated short hairpin (sh)RNA in the pancreatic cancer cell line Panc-1. The impact of of XIAP 
and survivin silencing on proliferation, invasion, migration, chemosensitivity and EMT was then evaluated.

\section{Materials and methods}

Materials. The Panc-1 pancreatic cancer cell line was purchased from the Cell Bank of Type Culture Collection (Shanghai Institute of Cell Biology, Chinese Academy of Sciences, Shanghai, China) and stored in liquid nitrogen at the Cell Bank of the State Key Laboratory of Medical Genetics (Changsha, China). When used, the cells were defrosted and revivified by incubating in a $37^{\circ} \mathrm{C}$ water bath for $\sim 1-2 \mathrm{~min}$, agitated for 1-2 min, then centrifuged at $1000 \mathrm{rpm}$ for $5 \mathrm{~min}$; cells were then resuspended in Dulbecco's modified Eagle's medium (DMEM) and fetal bovine serum (FBS), which were purchased from Invitrogen Life Technologies (Carlsbad, CA, USA), and $100 \mu \mathrm{g} / \mathrm{ml}$ streptomycin and $100 \mathrm{U} /$ ml penicillin (Gibco Life Technologies, Carlsbad, CA, USA), then stored at $37^{\circ} \mathrm{C}$ in a humidified atmosphere with $5 \% \mathrm{CO}_{2}$. XIAP-shRNA lentiviral vector $(\mathrm{LV}-\mathrm{X})$ and survivin-shRNA lentiviral vector (LV-S) were purchased from Genechem Corp. (Shanghai, China) and constructed as previously described (8). The sequences containing nonsense shRNA lentiviral vector ( $\mathrm{Lv}-\mathrm{Xnc}$ and $\mathrm{Lv}-\mathrm{Snc})$ were used as controls. MTT (tetrazolium salt) reagent was purchased from Sigma-Aldrich (St. Louis, MO, USA). The RevertAid First Strand cDNA Sysnthesis kit (cat. no. K1622) was purchased from Fermentas (Waltham, MA, USA). The SYBR TAQ real-time polymerase chain reaction (PCR) kit was purchased from Takara Bio, Inc. (Dalian, China). The Caspase-3/7 fluorescent enzyme activity detection kit was purchased from Promega Corp. (Madison, WI, USA). Rabbit anti-human polyclonal survivin primary antibodies were purchased from Novus Biologicals, LLC (NB500-201; Littleton, CO, USA) and goat anti-human polyclonal XIAP primary antibodies were products of R\&D Systems (AF8221; Minneapolis, MN, USA). Mouse anti-human monoclonal E-cadherin (sc-21791), mouse anti-human monoclonal Slug (sc-166476), mouse anti-human monoclonal phosphatase and tensin homolog (PTEN; sc-7974) and rabbit anti-human polyclonal phosphorylated (p)-Akt (Ser473; sc-33437) primary antibodies were purchased from Santa Cruz Biotechnology, Inc. (Dallas, TX, USA). Mouse anti-human $\beta$-actin primary antibodies (A1978) were purchased from Sigma-Aldrich. The corresponding rabbit anti-mouse (315-065-003), mouse anti-rabbit (211-065-109) and rabbit anti-goat (305-065-003) secondary antibodies were purchased from Jackson ImmunoResearch Laboratories, Inc. (West Grove, PA, USA). Matrige ${ }^{\circledR}$ and Transwell ${ }^{\circledR}$ chambers were purchased from BD Biosciences, (Franklin Lakes, NJ, USA). Puromycin and neomycin (G418) were purchased from Invitrogen Life Technologies.

Construction and infection of shRNA lentivirus. The construction of shRNA (shRNA) lentivirus targeting human XIAP and survivin was performed as described previously (8). Panc-1 cells $\left(1 \times 10^{5}\right)$ were seeded onto a six-well plate and continuously cultured with DMEM (containing $10 \% \mathrm{FBS}$ ) at $37^{\circ} \mathrm{C}$ and $5 \% \mathrm{CO}_{2}$ for $12 \mathrm{~h}$. Lentiviral infection was performed as previously described; in brief, the Panc-1 cells were first transfected with XIAP shRNA lentivirus (LV-X) and selected using puromycin; then, the stably XIAP shRNA-transfected cell clones were transfected with survivin shRNA lentivirus (LV-S) and selected using neomycin. Panc-1 cells transfected with XIAP and survivin shRNA were named Panc-1-XS, while Panc-1 cells transfected with nonsense XIAP and survivin shRNA were named Panc-1-XncSnc.

Quantitative PCR $(q P C R)$ and western blot analysis. Total RNA was extracted using TRIzol reagent (Invitrogen Life Technologies) according to the manufacturer's instructions. First strand cDNA synthesis was performed using the RevertAid First Strand cDNA Synthesis kit, as previously described. qPCR was performed using an ABI PRISM 7900 HT system (Applied Biosystems, Thermo Fisher Scientific, Waltham, MA, USA). PCR cycling condition were as follows: $95^{\circ} \mathrm{C}$ for $30 \mathrm{sec}$, followed by 40 cycles at $95^{\circ} \mathrm{C}$ for $5 \mathrm{sec}$ and $60^{\circ} \mathrm{C}$ for $30 \mathrm{sec}$. Gene expression was quantified using the comparative $\mathrm{Ct}$ method, by normalizing $\mathrm{Ct}$-values to a housekeeping gene ( $\beta$-actin) and calculating the relative expression values, as previously described (8). Primers used were identical to those used in a previous study (8). The total protein concentration was measured using a bicinchoninic acid protein quantification kit (Sigma-Aldrich, Taufkirchen, Germany). A total of $20 \mu \mathrm{g}$ protein was separated on $12 \%$ or $15 \%$ SDS-polyacrylamide gel and transferred to polyvinylidene fluoride membranes. Following blocking with blocking buffer [1X phosphate-buffered saline (PBS) containing $0.1 \%$ Tween-20 (PBST) and 5\% nonfat milk] for $45 \mathrm{~min}$, membranes were incubated with anti-XIAP $(1: 5,000)$, anti-survivin $(1: 2,000)$, anti-Slug $(1: 1,000)$, anti-PTEN $(1: 1,000)$ and anti-p-Akt $(1: 1,000)$ primary antibodies overnight at $4^{\circ} \mathrm{C}$. Following washing three times with 1X PBST for $10 \mathrm{~min}$, membranes were incubated with the corresponding rabbit anti-mouse, mouse anti-rabbit and rabbit anti-goat secondary antibodies (Jackson ImmunoResearch Laboratories, Inc. West Grove, PA, USA) at a 1:3,000 dilution ratio for $1 \mathrm{~h}$ at room temperature. Blots were washed to remove chemiluminescent substrate, then incubated in stripping buffer (Biosyntech, Inc., Beijing, China) for $5-15 \mathrm{~min}$ at $37^{\circ} \mathrm{C}$, followed by washing with $1 \mathrm{X}$ PBS for $5 \mathrm{~min}$ at room temperature. Following stripping, the membranes were reprobed with $\beta$-actin primary antibody $(1: 10,000)$ overnight at $4{ }^{\circ} \mathrm{C}$, followed by incubation with the corresponding rabbit anti-mouse secondary antibodies $(1: 10,000)$ for $1 \mathrm{~h}$ at room temperature. The immune reaction was visualized using enhanced chemiluminescence substrate (Western-Star ${ }^{\mathrm{TM}}$ Immunodetection System T1046; Life Technologies, Grand Island, NY, USA) and exposed to an autoradiograph film (X-OMAT AR, IB1651579; Kodak, Radnor, PA, USA), then developed using an X-OMAT $1000 \mathrm{~A}$ developer (Kodak). Bound proteins were visualized using ECL (Thermo Fisher Scientific) and detected using BioImaging Systems (BioDoc-It 220; UVP, Inc., Upland CA, USA).

Colony formation assay. $5 \times 10^{3}$ Panc-1-XS, Panc-1-XncSnc and non-transfected control Panc-1 cells were plated in 10-cm culture dishes. Following 14-21 days, cells were fixed with methanol and stained with $0.1 \%$ crystal violet, which were 
purchased from Chemical Reagent Factory of Hunan Normal University (Hunan, China). Colonies were counted by visual inspection. Planting efficiency (PE) and survival fraction (SF) were then calculated as follows: $\mathrm{PE}=$ (number of colonies formed/number of cells seeded)x $100 \%$; SF=number of colonies formed post treatment/(number of cells seeded x PE). This procedure was performed in triplicate.

MTT assay and cell apoptosis. Panc-1 cells were seeded onto 96-well plates and continuously cultured for time periods between 1 and 7 days in order to calculate the $\mathrm{IC}_{50}$ values. After cells attached, different concentrations of gemcitabine $(1,000$, $100,10,1,0.1,0.01$ and $0.001 \mu \mathrm{g} / \mathrm{ml})$ were added followed by incubation for the indicated times. Cells were then subjected to the MTT assay. In brief, $20 \mu 1 \mathrm{MTT}$ solution (5 mg/ml in PBS) was added to each well and incubated for $4 \mathrm{~h}$ at $37^{\circ} \mathrm{C}$. The cells were lysed in $100 \mu \mathrm{l}$ dimethyl sulfoxide (Beijing Dingguo Biotechnology Co., Ltd., Beijing, China) and analyzed on a ThermoMax microplate reader (Bio-Rad Laboratories Inc.) at a wavelength of $490 \mathrm{~nm}$. The growth curve was plotted and corresponding $\mathrm{IC}_{50}$ values were calculated using SPSS 15.0 software (SPSS, Inc., Chicago, IL, USA). Furthermore, in order to detect caspase-3/7 activity, cells were cultured in six-well plates and treated with gemcitabine $(0.5 \mathrm{mg} / \mathrm{l})$ for $24 \mathrm{~h}$. Caspase-3/7 activity was measured using a caspase-3/7 fluorescent enzyme activity detection kit. Caspase-3/-7 activity was evaluated using the Caspase-Glo®-3/-7 assay (Promega Corp.) according to the manufacturer's instructions. Luminescence was detected on a Sirius Luminometer and its software (version 3.2) (Berthold Inc., Germany) with $3 \mathrm{sec}$ delay and $10 \mathrm{sec}$ measurement. The caspase-3/-7 activity was normalized to the number of viable cells as determined by trypan blue staining Sigma-Aldrich (St. Louis, MO, USA), and the caspase 3/-7 fold induction by gemcitabine was determined by the ratio of caspase-3/-7 activity between the treated and control groups. To observe cell death, cells were cultured in 24-well plates and treated with gemcitabine $(0.5 \mu \mathrm{g} / \mathrm{ml})$ for $24 \mathrm{~h}$. Cells were then stained with Hoechst 33342 (5 $\mu \mathrm{g} / \mathrm{ml}$; BD Pharmingen, San Diego, CA, USA) for $5 \mathrm{~min}$ at $37^{\circ} \mathrm{C}$. Cells were washed and resuspended in PBS for morphological observation under a IX51 fluorescence microscope (Olympus Corp., Tokyo, Japan) with excitation at $355 \mathrm{~nm}$ and emission at $465 \mathrm{~nm}$. A minimum of 400 cells from six randomly selected fields per dish were counted and each experiment was performed in triplicate. The apoptotic index was calculated as previously described (14).

Wound healing assay and invasion assay. Panc-1 cells $\left(1 \times 10^{5}\right)$ were seeded onto a six-well plate and incubated until they reached $95 \%$ confluence. A wound was generated by scratching the surface of the plates with a pipette tip. Cells were then washed three times with PBS, incubated in serum-free DMEM for $48 \mathrm{~h}$ and then images were captured using an IX71 inverted microscope (Olympus Corp.). Invasion assays were performed using 24-well Matrigel ${ }^{\circledR}$-coated Transwells ${ }^{\circledR}$. Panc-1 cells were added to the upper chamber, which was coated with $75 \mu \mathrm{l} /$ well Matrigel $^{\circledR}$ and $0.6 \mathrm{ml} 10 \% \mathrm{FBS}-\mathrm{DMEM}$ was added to the lower chamber. Cells were incubated for $24 \mathrm{~h}$ at $37^{\circ} \mathrm{C}$ and $5 \% \mathrm{CO}_{2}$, and non-invading cells were removed with cotton swabs. Cells invading to the bottom of the membrane were fixed in
Table I. Inhibition rate of XIAP/survivin mRNA and protein expression in Panc-1-XS.

\begin{tabular}{lcc}
\hline & \multicolumn{2}{c}{ Inhibition rate (\%) } \\
\cline { 2 - 3 } Expression & XIAP & survivin \\
\hline mRNA & $54.62 \pm 1.45$ & $51.99 \pm 0.57$ \\
Protein & $47.19 \pm 3.13$ & $37.29 \pm 3.25$ \\
\hline
\end{tabular}

XIAP, X-linked inhibitor of apoptosis.

4\% paraformaldehyde (Chemical Reagent Factory of Hunan Normal University) and stained with $0.1 \%$ crystal violet for $30 \mathrm{~min}$ at $37^{\circ} \mathrm{C}$, washed with $\mathrm{PBS}$, and then counted in four different fields of vision under 40x magnification. Results are presented as the mean of three independent experiments.

Statistical analysis. All statistical analyses were performed using SPSS 15.0 software. All values are expressed as the mean \pm standard deviation. Student's t test or one-way analysis of variance were used to compare differences between groups. $\mathrm{P} \leq 0.05$ was considered to indicate a statistically significant difference between values.

\section{Results}

Simultaneous inhibition of XIAP and survivin expression in Panc-1 cells. As shown in Fig. 1, in Panc-1 cells stably expressing XIAP as well as survivin shRNA (Panc-1-XS), XIAP and survivin mRNA expression was significantly reduced by 54.62 and $51.99 \%$, respectively, compared with that in the Panc-1 and Panc-1-XncSnc cells $(\mathrm{P}<0.05)$. In addition, XIAP and survivin protein expression was reduced by 47.19 and $37.29 \%$, respectively, compared with that in the Panc-1-XncSnc cells $(\mathrm{P}<0.05)$ (Table I; Fig. 2). mRNA and protein expression in Panc-1-XncSnc cells showed no significant difference compared with that in the Panc-1 cells $(\mathrm{P}>0.05)$.

Detection of Panc-1 cell proliferation. Results showed that cell proliferation of Panc-1-XS cells was significantly inhibited compared with that of the Panc-1-XncSnc and Panc-1 cells $(\mathrm{P}<0.05)$ (Fig. 3). In addition, the colony formation rate of Panc-1-XS cells $(10.12 \pm 1.33 \%)$ was significantly reduced compared with that of the Panc-1-XncSnc cells (96.61 $\pm 7.89 \%)$

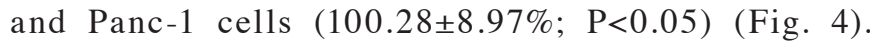
Proliferation of Panc-1-XncSnc cells showed no significant difference from that of Panc-1 cells $(\mathrm{P}>0.05)$.

Gemcitabine-induced activation of caspase-3/7, apoptosis and chemosensitivity are significantly enhanced in Panc-1-XS cells. Following treatment with various concentrations of gemcitabine $(0.001,0.01,0.1,1,10,100$ and $1000 \mathrm{mg} / \mathrm{ml})$, the relative activity of caspase-3/7 in Panc-1-XS cells was significantly increased to $15.02 \pm 0.57$ compared with that of Panc-1 cells and Panc-1-XncSnc cells $(8.87 \pm 0.19$ and 9.05 \pm 0.23 , respectively; $\mathrm{P}<0.05$; Fig. $5 \mathrm{~A})$. In addition, the rate of 

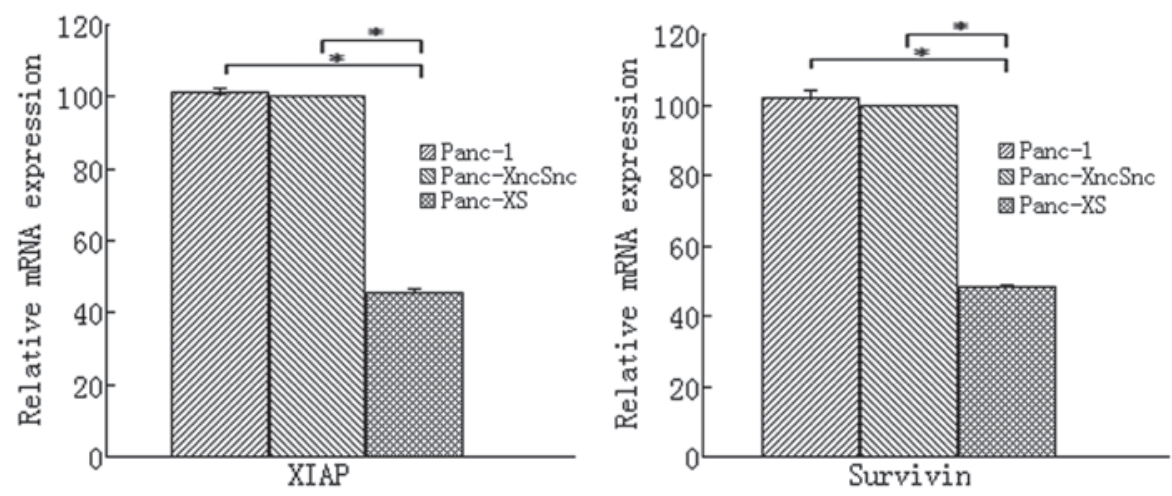

Figure 1. Relative expression of XIAP and survivin mRNA in Panc-1, Panc-1-XncSnc and Panc-1-XS cells. Values are presented as the mean \pm standard deviation ("P<0.05). XIAP, X-linked inhibitor of apoptosis; Panc-1-XS, Panc-1 cells transfected with XIAP and survivin short hairpin RNA; Panc-1-XncSnc, Panc-1 cells transfected with nonsense XIAP and survivin short hairpin RNA.

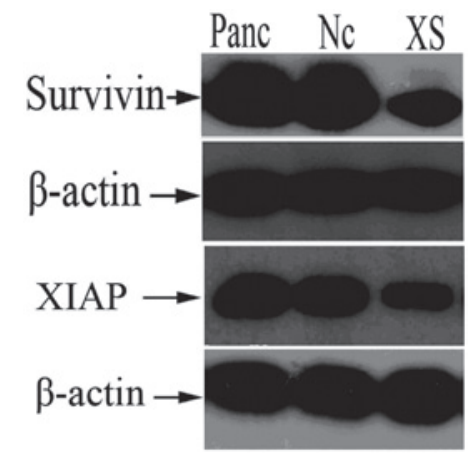

Figure 2. Protein expression of XIAP and survivin in Panc-1 (Panc), Panc-1-XncSnc (Nc) and Panc-1-XS (XS) cells. $\beta$-actin was used as an internal control. XIAP, X-linked inhibitor of apoptosis; Panc-1-XS, Panc-1 cells transfected with XIAP and survivin short hairpin RNA; Panc-1-XncSnc, Panc-1 cells transfected with nonsense XIAP and survivin short hairpin RNA.

apoptosis of Panc-1-XS cells (24.09 $\pm 2.75 \%)$ was significantly increased compared with that of Panc-1-XncSnc cells and Panc-1 cells $(12.068 \pm 1.22 \%$ and $12.09 \pm 1.97 \%$, respectively; $\mathrm{P}<0.05$; Fig. 5B). No significant difference was observed between Panc-1-XncSnc cells and Panc-1 cells $(\mathrm{P}>0.05$; Fig. 5B). Furthermore, the $\mathrm{IC}_{50}$-value of Panc-1-XS cells $(0.47 \pm 0.07 \mathrm{mg} / \mathrm{l})$ was significantly reduced compared with that of Panc-1-XncSnc cells and Panc-1 cells $(2.18 \pm 0.13$ and $2.13 \pm 0.18 \mathrm{mg} / 1$, respectively; $\mathrm{P}<0.05$; Fig. $5 \mathrm{C}$ ). Further testing showed that the $\mathrm{IC}_{50}$-value of Panc-1-XS cells was also reduced compared with that of Panc-1-X cells and Panc-1-S cells $(0.76 \pm 0.07 \mathrm{mg} / \mathrm{l}$ and $0.87 \pm 0.09 \mathrm{mg} / \mathrm{l}$, respectively; P<0.05; Fig. 5D and E).

Cell invasion and migration are significantly decreased in Panc-1-XS cells. The Transwell ${ }^{\circledR}$ chamber assay showed that the invasion of Panc-1-XS cells was significantly decreased compared with that of the Panc-1-XncSnc and Panc-1 cells $(\mathrm{P}<0.05)$ (Fig. 6). In addition, no significant differences were observed between the invasion of Panc-1-XncSnc cells and Panc-1 cells $(\mathrm{P}>0.05)$. The wound healing assay demonstrated that the wound healing capacity of Panc-1-XS cells was significantly decreased compared with that of the Panc-1-XncSnc and Panc-1 cells $(\mathrm{P}<0.05)$ (Fig. 7); however, no significant difference was observed between

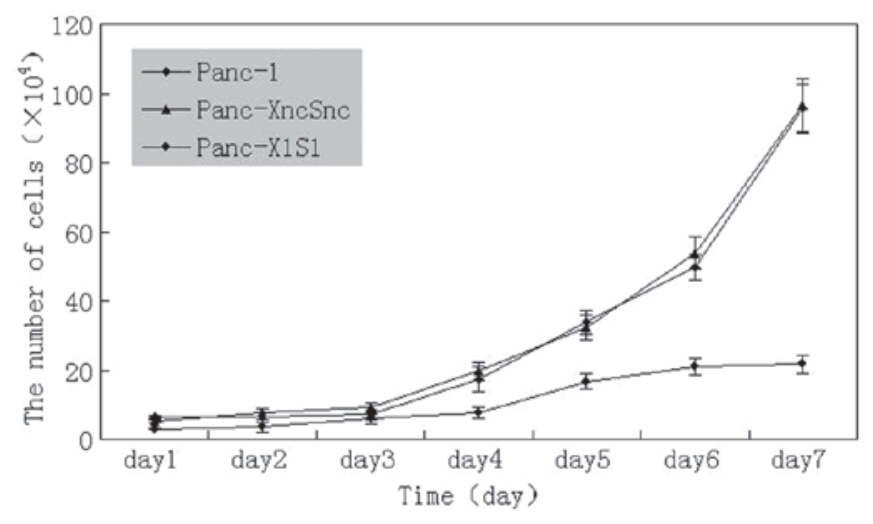

Figure 3. Cell growth curve of Panc-1, Panc-1-XncSnc and Panc-1-XS cells. XIAP, X-linked inhibitor of apoptosis; Panc-1-XS, Panc-1 cells transfected with XIAP and survivin short hairpin RNA; Panc-1-XncSnc, Panc-1 cells transfected with nonsense XIAP and survivin short hairpin RNA. Results are presented as the mean \pm standard deviation.

the wound healing capacity of Panc-1-XncSnc cells and Panc-1 cells $(\mathrm{P}>0.05)$.

Silencing of XIAP and survivin partially reverses the EMT, accompanied by increased protein expression of PTEN and decreased protein expression of $p$-Akt. In Panc-1-XS cells, protein expression of the epithelial marker E-cadherin was significantly increased, whereas the mesenchymal marker Slug was significantly reduced $(\mathrm{P}<0.05)$ (Fig. 8). These data indicated that simultaneous inhibition of the expression of XIAP and survivin may partially reverse the EMT in Panc-1 cells. Furthermore, the expression of PTEN protein was significantly increased in Panc-1-XS cells, while the p-Akt protein was significantly decreased compared with that in the Panc-1-XncSnc cells and Panc-1 cells $(\mathrm{P}<0.05)$ (Fig. 9). No significant differences were observed between the Panc-1-XncSnc and Panc-1 cells $(\mathrm{P}>0.05)$.

\section{Discussion}

Evasion of apoptosis is a well-known hallmark of numerous types of cancers (4). The IAP family has been shown to be particularly important in the regulation of apoptotic signaling, which results in the failure of cancer treatment in 


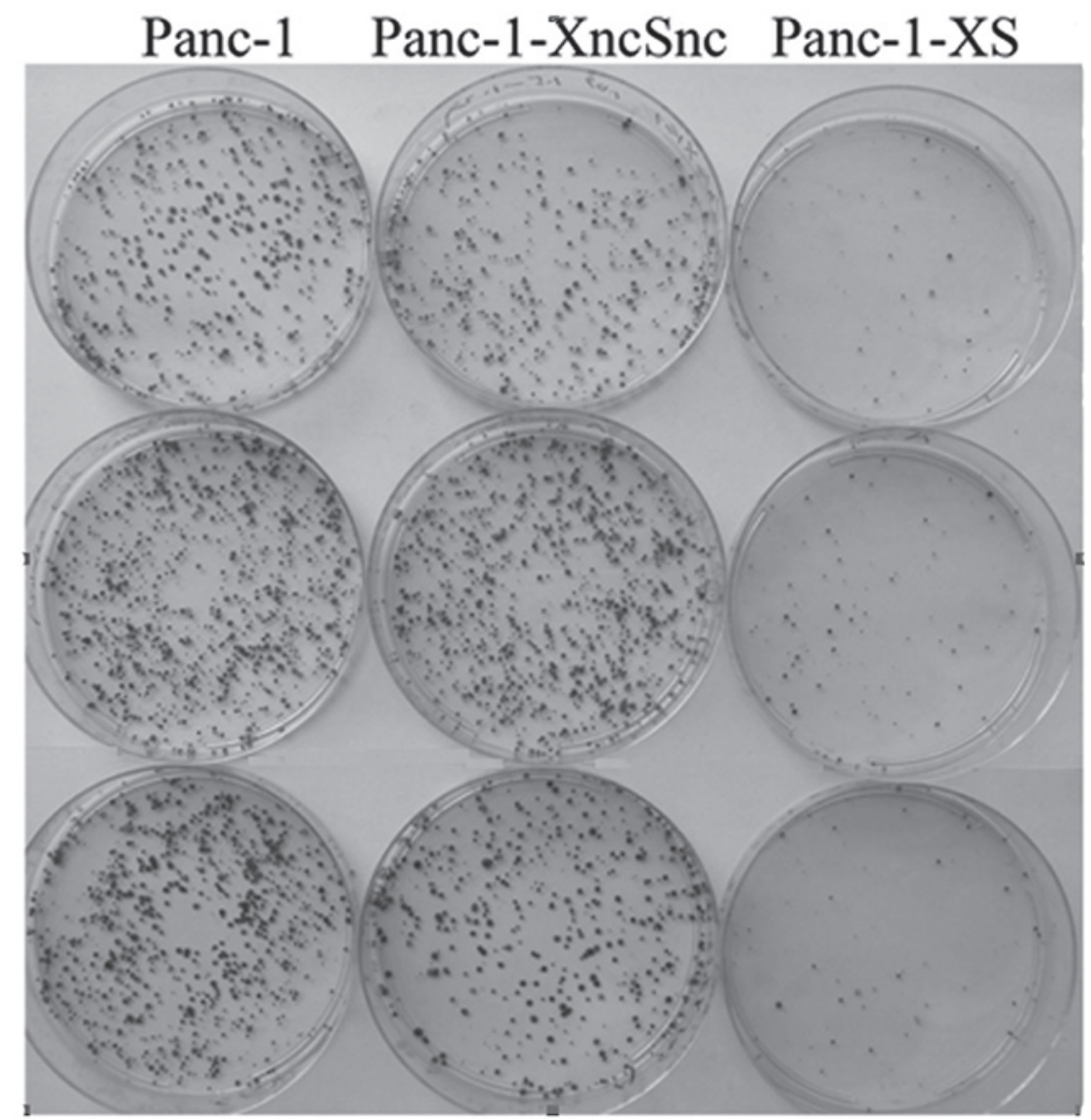

Figure 4. Cell colony formation of Panc-1, Panc-1-XncSnc and Panc-1-XS cells. XIAP, X-linked inhibitor of apoptosis; Panc-1-XS, Panc-1 cells transfected with XIAP and survivin short hairpin RNA; Panc-1-XncSnc, Panc-1 cells transfected with nonsense XIAP and survivin short hairpin RNA.
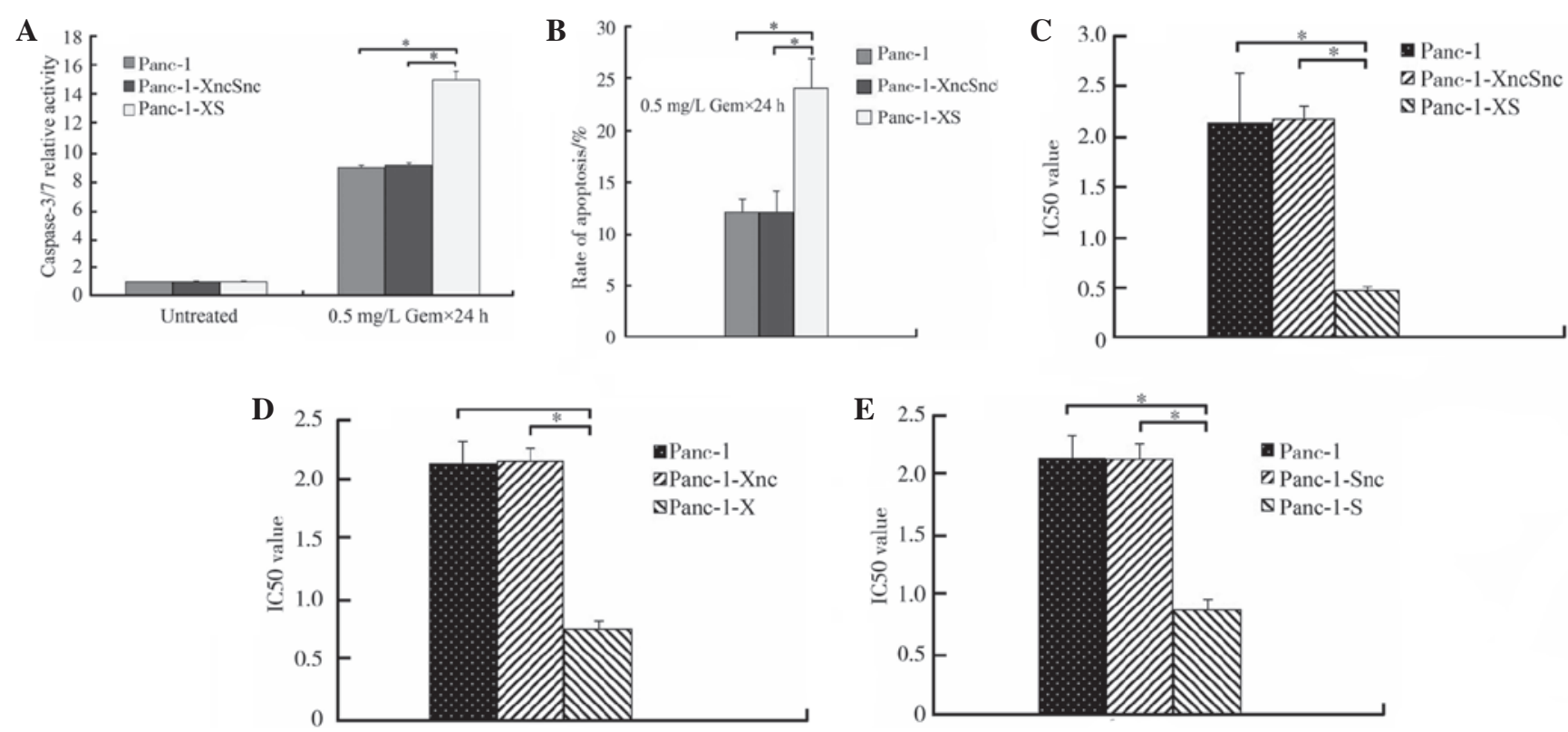

Figure 5. Gemcitabine induced activation of caspase 3/7, apoptosis and chemosensitivity are significantly enhanced in Panc-1-XS cells. (A) Gemcitabine induced caspase 3/7 relative activity. (B) Gemcitabine induced apoptosis. The $\mathrm{IC}_{50}$ value of (C) Panc-1-XS, (D) Panc-1-X and (E) Panc-1-S. XIAP, X-linked inhibitor of apoptosis; Panc-1-XS, Panc-1 cells transfected with XIAP and survivin short hairpin RNA; Panc-1-XncSnc, Panc-1 cells transfected with nonsense XIAP and survivin short hairpin RNA.

the clinic (15). XIAP and survivin are two important IAP family members and are frequently upregulated in numerous types of human tumor, including PC (15). Previous studies have shown that XIAP and survivin are highly expressed in PC and were closely associated with cell proliferation and sensitivity to chemotherapy with gemcitabine $(8,15,16)$. 

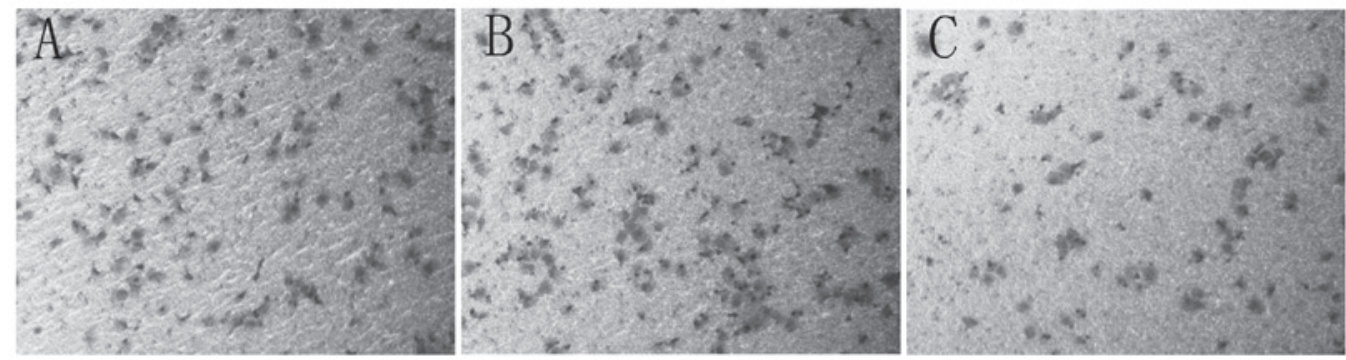

Figure 6. Effect of the simultaneous inhibition of XIAP and survivin in Panc-1 cells on cell invasion, as detected using the Transwell ${ }^{\circledR}$ chamber assay. (A) Untransfected Panc-1 cells; (B) Panc-1 cells transfected with nonsense XIAP and survivin short hairpin RNA; and (C) Panc-1 cells transfected with XIAP and survivin short hairpin RNA (magnification, $x 100$ ). XIAP, X-linked inhibitor of apoptosis.
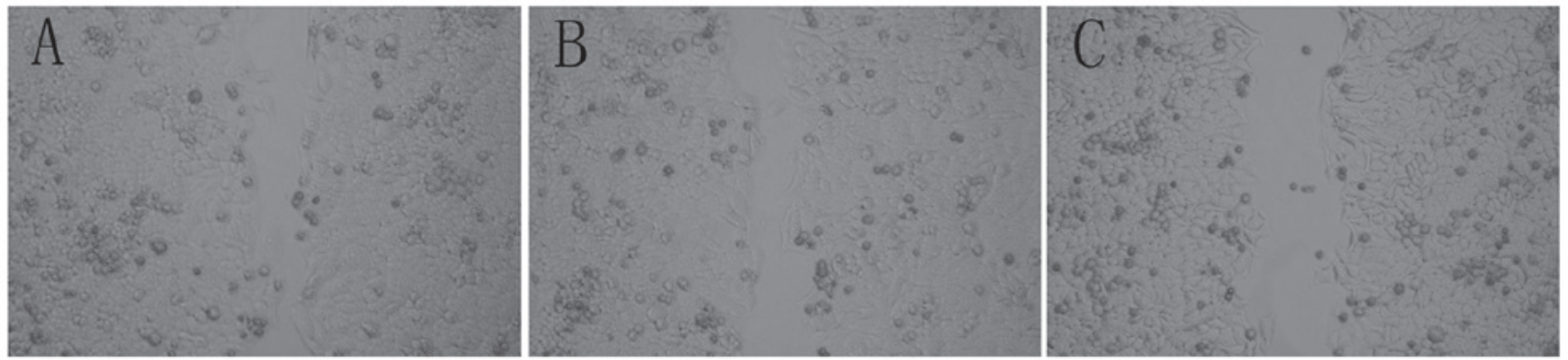

Figure 7. Effect of the simultaneous inhibition of XIAP and survivin in Panc-1 cells on cell migration, as detected using the wound healing assay. (A) Untransfected Panc-1 cells; (B) Panc-1 cells transfected with nonsense XIAP and survivin short hairpin RNA; and (C) Panc-1 cells transfected with XIAP and survivin short hairpin RNA (magnification, $\mathrm{x} 40$ ). XIAP, X-linked inhibitor of apoptosis.

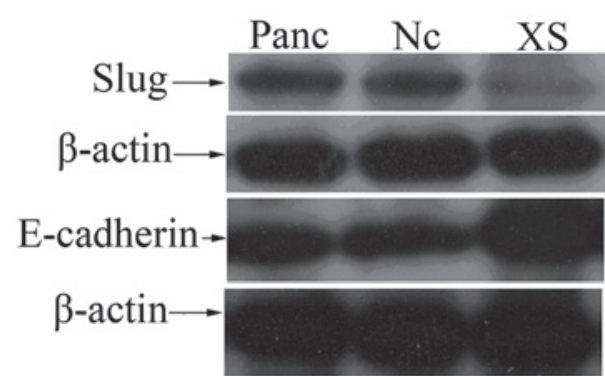

Figure 8. Effect of the simultaneous inhibition of XIAP and survivin in Panc-1 cells on the epithelial-mesenchymal transition of Panc-1 cells. Protein expression was determined for the epithelial marker E-cadherin and the mesenchymal marker Slug. Panc, untransfected Panc-1 cells; Nc, Panc-1 cells transfected with nonsense XIAP and survivin short hairpin RNA; XS, Panc-1 cells transfected with XIAP and survivin short hairpin RNA. $\beta$-actin was used as an internal control. XIAP, X-linked inhibitor of apoptosis.

Therefore, these two genes represent interesting candidates for a target-directed molecular-based anti-tumor therapy.

The EMT is characterized by the loss of cell-to-cell adhesion and a phenotypic change from an epithelial morphology to a fibroblast-like motile morphology (10). The hallmark of EMT is the loss of the epithelial adhesion molecule E-cadherin and gain of mesenchymal cell markers, including Slug and Snail (12). In addition, EMT has been reported to have an important role in cancer cell invasion and metastasis (17). Numerous studies have shown that PC cells which have acquired a mesenchymal phenotype through EMT are usually gemcitabine-resistant (18). Therefore, EMT has also been considered as one of the important mechanisms of drug resistance in PC. However, the molecular mechanisms

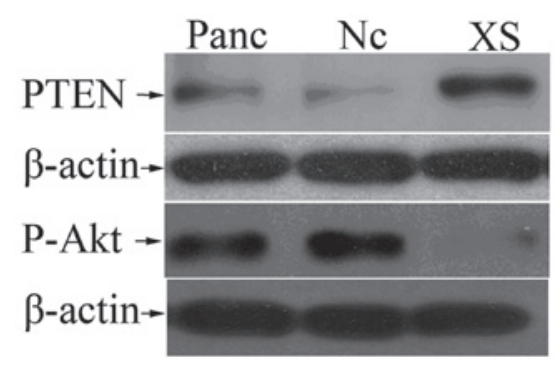

Figure 9. Effect of the simultaneous inhibition of XIAP and survivin in Panc-1 cells on PTEN and p-Akt protein expression levels. Panc, Untransfected Panc-1 cells; Nc, Panc-1 cells transfected with nonsense XIAP and survivin short hairpin RNA; XS, Panc-1 cells transfected with XIAP and survivin short hairpin RNA. $\beta$-actin was used as an internal control. XIAP, $\mathrm{X}$-linked inhibitor of apoptosis; PTEN, phosphatase and tensin homolog; $\mathrm{p}-$, phosphorylated.

underlying EMT and gemcitabine resistance have remained to be elucidated.

RNAi technology is widely used in the study of gene function and is superior to the majority of alternative gene knockout technologies. Lentiviral vectors enable the efficient, stable transfection of target cells and are able to be integrated into the target cell genome in order to achieve the sustained expression of carried genes (19). Lentiviral vector-mediated RNAi technology has been tested for gene targeting treatment studies in several types of tumor (20-22). In the present study, lentiviral vectors stably expressing shRNA targeting the XIAP and survivin genes were constructed and transfected into Panc-1 cells. Simultaneous silencing of XIAP and survivin expression significantly inhibited cell proliferation, increased caspase-3/7 activity and increased sensitivity to gemcitabine, 
which was consistent with previous reports using other tumor cell types $(21,23)$. Of note, a novel phenomenon was identified in the present study, namely the partial inversion of the EMT following simultaneous inhibition of the expression of XIAP and survivin in Panc-1 cells; this process is known as mesenchymal-epithelial transition (MET). MET was indicated by the significant upregulation of the protein expression of E-cadherin and significant downregulation of Slug expression. In addition, MET was accompanied by significantly reduced cell invasion and migration. To the best of our knowledge, the present study was the first to report an association between IAPs, including XIAP and survivin, and EMT in PC; however, the exact mechanism of this interaction remains to be elucidated.

As mentioned above, PC cells undergo EMT at early stages. EMT is a key step in tumor progression as it induces increased motility, invasion and chemoresistance in PC cells (11). The results of the present study demonstrated for the first time, to the best of our knowledge, that downregulation of XIAP and survivin resulted in the gain of epithelial markers and the loss of mesenchymal markers, suggesting that elevated XIAP and survivin expression may induce EMT and therefore have an important role in the regulation of motility, invasiveness and metastatic potential in PC cells. However, the mechanisms underlying the involvement of XIAP and survivin in EMT require further exploration.

Previous studies have shown that numerous molecular pathways, including Notch and nuclear factor- $\kappa \mathrm{B}$ may have important roles in the EMT process $(18,24)$. The PTEN/phosphatidylinositol 3-kinase (PI3K)/Akt signaling pathway regulates oncogene activation through inducing the localization of Akt to the cell membrane where it activates numerous downstream key cellular processes, including glucose metabolism, cell proliferation, apoptosis, transcription and cell migration (25-27). The PTEN/PI3K/Akt signaling pathway was found to be deregulated in PC due to the irregular activation of the PI3K/Akt signaling pathway and downregulation or deletion of PTEN; this was suggested to have important roles in the occurrence, development and gemcitabine resistance of PC (28-30). In the present study, it was demonstrated that Panc-1-XS cells exhibited a significant upregulation of PTEN and downregulation of $\mathrm{p}-\mathrm{Akt}$ protein levels. It was therefore speculated that simultaneous inhibition of the expression of XIAP and survivin resulted in upregulated expression of PTEN protein, which led to decreased p-Akt protein expression and mediated the partial reversion of the EMT phenotype (MET); therefore, in turn, MET ultimately resulted in decreased invasion and metastasis as well as increased chemosensitivity to gemcitabine in PC cells.

In conclusion, the results of the present study demonstrated that the simultaneous inhibition of XIAP and survivin expression significantly inhibited proliferation and enhanced apoptosis in Panc-1 cells. In addition, inhibiting the expression of XIAP and survivin in Panc-1 cells reversed the EMT and resulted in increased chemosensitivity as well as reduced cell invasion and migration. Furthermore, it was hypothesized that the PTEN/PI3K/Akt pathway may have important roles in this process. The exploration experiments performed in the present study were a preliminary approach and a more in-depth investigation is required in order to confirm these results.

\section{Acknowledgements}

The present study was supported by a grant from the Freedom Exploration Program of Central South University (grant no. 2011QNZT153).

\section{References}

1. Siegel R, Naishadham D and Jemal A: Cancer statistics, 2013. CA Cancer J Clin 63: 11-30, 2013.

2. Hidalgo M: Pancreatic cancer. $N$ Engl J Med 362: 1605-1710, 2010

3. Muniraj T, Jamidar PA and Aslanian HR: Pancreatic cancer: a comprehensive review and update. Dis Mon 59: 368-402, 2013.

4. Hanahan D and Weinberg RA: Hallmarks of cancer: the next generation. Cell 144: 646-674, 2011.

5. Wei Y, Fan T and Yu M: Inhibitor of apoptosis proteins and apoptosis. Acta Biochim Biophys Sin (Shanghai) 40: 278-288, 2008.

6. Silke J and Meier P: Inhibitor of apoptosis (IAP) proteins-modulators of cell death and inflammation. Cold Spring Harb Perspect Biol 5, 2013.

7. Mehrotra S, Languino LR, Raskett CM, et al: IAP regulation of metastasis. Cancer Cell 17: 53-64, 2010.

8. Jiang C, Tan T, Yi XP, et al: Lentivirus-mediated shRNA targeting XIAP and survivin inhibit SW1990 pancreatic cancer cell proliferation in vitro and in vivo. Mol Med Rep 4: 667-674, 2011.

9. Krepela E, Dankova P, Moravcikova E, et al: Increased expression of inhibitor of apoptosis proteins, survivin and XIAP, in non-small cell lung carcinoma. Int J Oncol 35: 1449-1462, 2009.

10. Kalluri R and Weinberg RA: The basics of epithelial-mesenchymal transition. J Clin Invest 119: 1420-1428, 2009.

11. Arumugam T, Ramachandran V, Fournier KF, et al: Epithelial to mesenchymal transition contributes to drug resistance in pancreatic cancer. Cancer Res 69: 5820-5828, 2009.

12. Iwatsuki M,Mimori K, Yokobori T, et al: Epithelial-mesenchymal transition in cancer development and its clinical significance. Cancer Sci 101: 293-299, 2010.

13. Hannon GJ: RNA interference. Nature 418: 244-251, 2002.

14. Tonomura H, Takahashi KA, Mazda O, et al: Glutamine protects articular chondrocytes from heat stress and NO-induced apoptosis with HSP70 expression. Osteoarthritis Cartilage 14: 545-553, 2006.

15. LaCasse EC, Mahoney DJ, Cheung HH, et al: IAP-targeted therapies for cancer. Oncogene 27: 6252-6275, 2008.

16. Urrutia, R: The IAP: more international than ever. Pancreatology 9: III-IV, 2009.

17. Thiery JP, Acloque H, Huang RYJ and Nieto MA: Epithelial-mesenchymal transitions in development and disease. Cell 139: 871-890, 2009.

18. Wang Z, Li Y, Kong D, et al: Acquisition of epithelial-mesenchymal transition phenotype of gemcitabine-resistant pancreatic cancer cells is linked with activation of the notch signaling pathway. Cancer Res 69: 2400-2407, 2009.

19. Deng Y, Wang CC, Choy KW, et al: Therapeutic potentials of gene silencing by RNA interference: Principles, challenges, and new strategies. Gene 538: 217-227, 2014.

20. Jiang G, Li J, Zeng Z and Xian L. Lentivirus-mediated gene therapy by suppressing survivin in BALB/c nude mice bearing oral squamous cell carcinoma. Cancer Biol Ther 5: 435-440, 2006.

21. Kunze D, Kraemer K, Erdmann K, et al: Simultaneous siRNA-mediated knockdown of antiapoptotic BCL2, Bcl-xL, XIAP and survivin in bladder cancer cells. Int J Oncol 41: 1271-1277, 2012.

22. Ravet E, Lulka H, Gross F, et al: Using lentiviral vectors for efficient pancreatic cancer gene therapy. Cancer Gene Ther 17: 315-324, 2010.

23. Ruckert F, Samm N, Lehner AK, et al: Simultaneous gene silencing of Bcl-2, XIAP and Survivin re-sensitizes pancreatic cancer cells towards apoptosis. Bmc Cancer 10: 379, 2010.

24. Min C, Eddy SF, Sherr DH, et al: NF- $\kappa \mathrm{B}$ and epithelial to mesenchymal transition of cancer. J Cell Biochem 104: 733-744, 2008. 
25. Carnero A, Blanco-Aparicio C, Renner O, et al: The PTEN/PI3K/AKT signalling pathway in cancer, therapeutic implications. Curr Cancer Drug Targets 8: 187-198, 2008.

26. Jiang $\mathrm{BH}$ and Liu LZ: PI3K/PTEN signaling in angiogenesis and tumorigenesis. Adv Cancer Res 102: 19-65, 2009.

27. Hafsi S, Pezzino FM, Candido S, et al: Gene alterations in the $\mathrm{PI} 3 \mathrm{~K} / \mathrm{PTEN} / \mathrm{AKT}$ pathway as a mechanism of drug-resistance (review). Int J Oncol 40: 639-644, 2012.
28. Ma J, Sawai H, Ochi N, et al: PTEN regulates angiogenesis through PI3K/Akt/VEGF signaling pathway in human pancreatic cancer cells. Mol Cell Biochem 331: 161-171, 2009.

29. Chen X, Liao J, Lu Y, et al: Activation of the PI3K/Akt pathway mediates bone morphogenetic protein 2-induced invasion of pancreatic cancer cells Panc-1. Pathol Oncol Res 17: 257-261, 2011.

30. Parsons CM, Muilenburg D, Bowles TL, et al: The role of Akt activation in the response to chemotherapy in pancreatic cancer. Anticancer Res 30: 3279-3289, 2010. 